\title{
R1 Cooling Gel Plus R2 Moisturizing/Sun Protecting Lotion
}

National Cancer Institute

\section{Source}

National Cancer Institute. R1 Cooling Gel Plus R2 Moisturizing/Sun Protecting Lotion. NCI

Thesaurus. Code C102555.

A skin care treatment system consisting of a cytokine-containing milk-based cooling gel and a cytokine-containing milk-based soothing lotion with hydrating and potential protective activity against radiation-induced dermatitis. Application of the R1 cooling gel immediately upon radiation treatment may hydrate the skin, provide cooling relief, and may protect the skin against radiation-induced dermatitis. The subsequent application of the R2 lotion moisturizes the skin while also protecting the skin against inflammation. In addition, R2 contains avobenzone, homosalate, octinoxate and octocrylene, which exert UVA and UVB skin protective activity. 